\title{
Reação do mercado ao ingresso (saída) ao ISE: estudo de evento e análise da liquidez
}

\section{Market reaction to entry (exit) to the ISE: event study and liquidity analysis}

\author{
DOI: $10.46814 /$ lajdv3n3-025
}

Recebimento dos originais: 01/052021

Aceitação para publicação: 31/06/2021

\author{
Maximiliano Kruel \\ Mestre em Administração. Universidade de Salamanca, Calle Espejo n ${ }^{\circ}$ 2, Salamanca, Espanha. CEP \\ 37007. \\ E-mail:maxkruel@hotmail.com
}

\author{
Kelmara Mendes Vieira \\ Doutora em Administração. Universidade Federal de Santa Maria, Rua Roraima nº 1000, Santa \\ Maria/RS, Brasil. CEP 97105-900. \\ E-mail: kelmara@smail.ufsm.br
}

\section{RESUMO}

O presente trabalho analisa a reação dos investidores quanto à entrada (saída) das ações das empresas que aderem ao Índice de Sustentabilidade Empresarial (ISE) da BOVESPA para os anos de 2005-2009, e busca identificar possíveis alterações nos índices de liquidez de algumas variáveis. Através da metodologia de estudo de evento e teste da influência da liquidez de mercado, o trabalho busca evidenciar a performance através da eficiência de mercado sob a forma semi-forte. Foram selecionados cinco indicadores de liquidez para identificar as diferenças no ingresso (saída) do ISE. Os resultados demonstram que mesmo as informações sendo anteriormente publicadas quanto ao ingresso (saída) do índice ISE, não há evidencia de retornos acima dos esperados, apesar de haver existência de alguns resíduos, positivos e negativos, em alguns instantes anteriores e posteriores à data do evento. Para os testes de liquidez, foram encontradas relações apenas na amostra completa das ações das empresas, já na amostra de ingresso (saída), pode-se apenas verificar relação com o retorno dos índices, BOVESPA e FGV 100. Desta forma, segundo a teoria de eficiência de mercado na forma semi-forte, as práticas das empresas que fazem parte do ISE já estão presentes nos preços das ações.

Palavras-chave: Índice de sustentabilidade, liquidez, mercado de capitais brasileiro.

\begin{abstract}
The present work analyzes entrance (exit) investor's reaction of the companies' assets who adhere to the Enterprise Sustainability Index (ISE) of the BOVESPA, between 2005-2009, and search to identify possible alterations in the liquidity ratios for some variables. Through the event study methodology and the influence of market liquidity test, this paper brings evidence of market performance throughout semi strong market efficiency. Five liquidity indicators had been selected to identify the differences in the ingression (exit) of the ISE. The results demonstrate that even information have being previously published of the ingression (exit) ISE index, it does not have returns above of the waited ones, although there are existence of bigger, positive and negative average residues, in some instants, previous and subsequent of the event date. The liquidity tests had been found relations only for complete samples of the companies' assets. The ingression (exit) can only be verified relation with the return of the index, BOVESPA and FGV 100. In such a way, the Efficiency of Market Theory, in the semi strong form, the companies' actions of the ISE are already presents in their assets prices.
\end{abstract}


Keywords: Sustainability index, liquidity, Brazilian capital market.

\section{INTRODUÇÃO}

O crescimento exponencial da população, do consumo de recursos naturais, do capital e da poluição segue no planeta, podendo exceder rapidamente os limites sustentáveis de promoção da vida na terra. Os materiais e energia utilizados pela população e estrutura do capital não surgem do nada, são extraídos do planeta e, não desaparecem. Fontes e sumidouros são imprescindíveis para sugar os diversos resíduos e poluentes criados na economia global. Acontece que hoje o planeta já sofre um overshoot (exceder, ir longe demais, ultrapassar limites) de crescimento acelerado, ultrapassando barreiras do qual o sistema, talvez, não possa seguir com segurança (MEADOWS, RANDERS e MEADOWS, 2007).

Demonstrar o impacto que a política de sustentabilidade poderá trazer para a organização para ganhar reconhecimento do público à custa do dinheiro dos acionistas, ou se efetivamente a empresa traz ganhos maiores quando adota tal postura no mercado sobre os efeitos a entrada das empresas com ações no Índice de Sustentabilidade Empresarial, ISE, cria um cenário propício para testar a hipótese de eficiência informacional na forma semi-forte ${ }^{1}$, do mercado brasileiro.

A responsabilidade social e ambiental envolve uma série de ações que potencialmente aumentam os custos das organizações, como por exemplo, a melhoria das condições de trabalho e processos produtivos com menos impacto ambiental. Estes custos devem ser compensados com benefícios que possam ser economicamente sustentáveis (BECCHETTI, DI GIACOMO e PINNACCHIO, 2005).

Segundo Finch, 2006, os índices de sustentabilidade têm por objetivo prover um benchmark (busca das melhores práticas na indústria que conduzem ao desempenho superior) de performance financeira das companhias e ajudar os investidores que buscam empresas listadas em práticas de trabalho sustentáveis e que incorporam desenvolvimento socioambientais.

Estar listada nos índices de sustentabilidade remete as empresas a um nível superior de credibilidade, sendo bem vista perante gestores de carteiras de fundos éticos, que promovem atenção dos investidores para empresas de Responsabilidade Social Corporativa, conseqüentemente traz valor para os interesses dos shareholders (acionista ou proprietário da ação), buscando vantagens competitivas de mercado (HILEMAN, BESLY e SAVITZ, 2006).

\footnotetext{
${ }^{1}$ Proposta por FAMA (1991), a hipótese de eficiência de mercado na forma semi-forte afirma que os preços refletem toda a informação publicamente disponível, ou seja, o investidor não pode obter excessos de retorno utilizando-se de informações publicamente disponíveis.
} 
Empresas que adotam políticas sustentáveis seriam recompensadas com um custo de capital menor e haveria um aumento da demanda pelos ativos, levando assim a um maior preço de mercado. Num estudo para empresas não financeiras brasileiras listadas na Bolsa de Valores de São Paulo (BOVESPA), entre os anos 2005 e 2007, Rossi Jr., 2009, encontrou melhores performances em empresas que participam do Índice de Sustentabilidade Empresarial (ISE).

O Índice de Sustentabilidade Empresarial (ISE) criado em 2005 pela Bolsa de Valores de São Paulo - BOVESPA conjuntamente com outras entidades, tem por objetivo refletir o retorno de uma carteira composta por ações de empresas com reconhecido comprometimento com a responsabilidade social e a sustentabilidade empresarial, e também atuar como promotor das boas práticas no meio empresarial brasileiro (BOVESPA, 2008).

Além de promover a visibilidade das companhias que conquistam o posto de "elegíveis" da carteira de índices, uma empresa que chega lá ganha, automaticamente, o prestígio de fazer parte de uma lista composta por instituições que seguem à risca as melhores práticas de transparência, credibilidade, disciplina e comprometimento com o seu negócio, acompanhado por analistas e investidores, resultando num aumento da liquidez e maior acesso a novas distribuições públicas (BM\&FBOVESPA, 2009).

Se a adição de uma empresa num índice é associada a uma melhora na performance corporativa, este progresso deverá resultar imediatamente num crescimento da demanda por parte dos investidores sobre esta empresa, especulando sobre uma expectativa futura acerca de sua performance financeira (DENIS, MCCONNELL, OVTCHINNIKOV e YU, 2001). Neste sentido a entrada e saída do índice podem afetar a performance de mercado de diversas maneiras, existindo diferentes proxies (variáveis explicativas) para analisar o movimento dos preços das ações, como: os retornos anormais e a liquidez de mercado (MEDEIROS e SILVEIRA, 2007, CHEUNG, 2009).

A identificação de anomalias no período de entrada (saída) das empresas no Índice de Sustentabilidade Empresarial (ISE) comprovaria que através de informações publicamente disponíveis, os investidores estariam obtendo ganhos extras, o que levaria à rejeição de tal hipótese. Por outro lado, caso não se verifique a existência de retornos anormais significativos, a hipótese de eficiência semiforte seria aceita, indicando que, para os investidores brasileiros, o ingresso (entrada/saída) de empresas com ações no Índice de Sustentabilidade Empresarial (ISE) não traz nenhuma anomalia de mercado.

Assim aproveitando o importante cenário desenhado pelo mercado de capitais brasileiro, este trabalho tem por objetivo analisar a reação dos investidores à entrada (saída) do Índice de Sustentabilidade Empresarial (ISE) e a liquidez das ações das empresas que ingressaram (saíram) no índice ISE. 
Este artigo foi estruturado de acordo com a seguinte forma: após a introdução, o item 2 será dedicado ao referencial teórico, o item 3 apresenta a metodologia adotada nesta pesquisa e no item 4 os resultados obtidos. No item 5 apresenta as conclusões do trabalho, suas limitações e propostas para futuras pesquisas que possam usar sob diferentes óticas afim de obter outras evidencias.

\section{REFERENCIAL TEÓRICO}

\subsection{RELAÇÃO ENTRE RESPONSABILIDADE SOCIAL E PERFORMANCE FINANCEIRA}

Desde que começaram as preocupações com responsabilidade social, existe também um desconforto acerca do resultado econômico-financeiro, ou seja, de que maneira poderá ser obtido o investimento aplicado nesta área. Isto porque, além das questões ambientais e legislações em que estão inseridas, as empresas precisam saber quanto vai custar e qual o retorno esperado destes investimentos.

Deixando a parte de criar lucro, companhias sustentáveis são líderes em capturar outros critérios não financeiros com o intuito de melhorar performance, como: gerenciamento de qualidade, estrutura de governança corporativa, reputação, gerenciamento de capital humano, relacionamento com stakeholders, proteção ao meio-ambiente e responsabilidade social corporativa. Contrariando os negócios tradicionais que visam o lucro sem importar-se com as conseqüências socioambientais, um novo estilo de investimento ganha mercado chamando atenção dos investidores, criando valor para as empresas, chamado de investimentos éticos (LO e SHEU, 2007).

Algumas formas de se evidenciar o aumento do valor de uma empresa em função dos investimentos socioambientais pode ser a possibilidade da empresa diferenciar seus produtos em seu mercado, diminui o risco da empresa sofrer multas governamentais e também pode diminuir a exposição da empresa ao risco. Todo o esforço para descobrir como atividades socialmente responsáveis podem aumentar o valor de uma empresa é problemático (MACKEY, MACKEY e BARNEY, 2005). Os resultados conflitantes na relação entre performance financeira e investimentos socioambientais encontrados em estudos anteriores talvez sejam devido a diferentes estágios de influência destas duas variáveis (RODGERS, CHOY e GUIRAL, 2008).

Makni, Francoeur e Bellavance (2008) não encontram relação entre performance financeira e investimentos socioambientais, mas verificam uma relação negativa entre performance financeira e investimentos sociais internos: maiores são os investimentos sociais internos, menores são os níveis de perfomance financeira.

Por outro lado os trabalhos de Moskowitz (1972), Parker e Eilbirt (1975), Preston (1978), Ullmann (1985), McGuide, Sundgren e Scheneeweis (1988), Waddock e Graves (1997), encontraram relações positivas entre performance financeira e performance sociais corporativas (CESAR e JÚNIOR, 2008). 
Diferenciando dos demais, Borba (2005) encontrou relações nulas para indicadores financeiros contábeis e desempenho social, porém quando ocorreram relações significativas das variáveis no modelo, houve relações positivas. Neste trabalho, Borba (2005) utilizou o Valor da Firma proposto pelo Economática ${ }^{\circledR}$ e o Q de Tobim para variáveis de performance financeira a valores de mercado e Retorno do Lucro Operacional (LOAT), Retorno da Geração Bruta de Caixa (LAJIRDA) e Retorno do Lucro Operacional Próprio (LOPAT) para desempenho econômico financeiro contábil.

Um recente estudo com dados em painel no Brasil destaca-se o trabalho de Cézar e Junior (2008), pela qual busca encontrar relação entre performance financeira e investimentos socioambientais. Este estudo investiga a relação entre a performance social e ambiental e a performance financeira das empresas com ações negociadas na Bolsa de Valores de São Paulo durante o período compreendido entre 1999-2006, no qual analisada pela metodologia de dados em painel. Como resultado os autores evidenciaram a relação negativa entre ROA (retorno sobre os ativos) e os indicadores sociais internos, não encontrando relações significativas entre as outras variáveis.

\subsection{INGRESSO (SAÍDA) DOS ÍNDICES DE MERCADO}

Tradicionalmente a teoria financeira assume que a inclusão ou exclusão de uma ação a algum índice financeiro não deveria afetar seu respectivo preço. Alguns estudos sugerem que o mercado reage para refletir uma mudança de posição dos investidores em relação ao fluxo de caixa esperado ou ao custo de capital da empresa. Outros estudos levam em consideração que a demanda em relação ao preço é perfeitamente elástica. A explicação para que este movimento ocorra é que o preço é afetado por uma demanda anormal para esta ação.

Arantes (2006) buscou identificar a existência de diferença entre o desempenho econômico de empresas que possuem investimentos em responsabilidade social e o desempenho econômico de empresas que não possuem tal investimento. O autor fez uma comparação da evolução no valor das ações negociadas em Nova Iorque e em São Paulo, concluindo que empresas que apresentam investimentos em responsabilidade social têm suas ações com valorizações acima das que não possuem esse tipo de investimento.

As evidências desse estudo são confirmadas pelo premiado trabalho no $9^{\circ}$ Encontro Brasileiro de Finanças de Rossi Jr. (2009) sobre uma resposta ao valor da empresa ao índice de sustentabilidade brasileira para companhias não financeiras listadas na bolsa de valores de São Paulo no período de 2005-2007. Este estudo usa como proxy (variável de análise) de cálculo para o valor da firma o Q de Tobin, definido como a razão do valor da firma pela valorização dos seus ativos, por empresas para cada final do ano fiscal. Conclui que empresas brasileiras têm um ganho significativo adotando políticas de responsabilidade social, tendo as ações destas empresas com participação no ISE um 
prêmio comparado às ações de outras firmas, gerando valor às empresas. O resultado desta estimação confirma o estudo de Lo e Sheu (2007) para o mercado americano com as empresas não financeiras pertencentes ao Dow Jones Sustainability índex no período de 1999-2002. Usando o Q de Tobin como proxy do valor da firma foi encontrada relação positiva entre sustentabilidade corporativa e valor de mercado.

Dias (2007) por outro lado, evidenciou através de um estudo de evento os retornos anormais dos preços das ações de empresas que ingressaram na carteira do ISE nos anos 2006 e 2007. As análises foram estudadas de forma distintas, investigadas através da hipótese da existência de retornos anormais acumulados, as amostras foram constituídas pelos valores das ações de empresas pertencentes às carteiras do índice nos anos 2006 e 2007, confrontadas com o índice do mercado Ibovespa do mesmo ano. Também foram analisadas carteiras de empresas que não aderiram aos níveis de governança corporativa diferenciados da BOVESPA (nível 2 e Novo Mercado) e análises excluindo as instituições financeiras (por apresentarem as maiores valorizações das carteiras do ISE).

Sob a ótica de performance corporativa e desenvolvimento sustentável, o estudo de López, Garcia e Rodriguez (2007) analisam indicadores de sustentabilidade para uma amostra de dois grupos de empresas totalizando 110 firmas com similaridade em tamanho, estrutura de capital e segmento de atividade para o período de 1998-2004. O primeiro grupo composto por 55 empresas européias que foram incluídas no índice Dow Jones Sustainability Index (DJSI) e o segundo grupo formado por 55 empresas européias incluídas no Dow Jones Global Index (DJGI) no mesmo período, porém que não tenha sido incluída no índice de sustentabilidade (DJSI).

Para uma amostra de empresas que anunciaram ingresso (entrada/saída) no período de 2002 a 2006 no estudo de Tsai (2007) e no período de 2002-2007 no estudo de Karlsson e Chakarova (2008), foram examinados o impacto da inclusão (entrada) e exclusão (saída) das ações de empresas dos índices Dow Jones Sustainability World Index (DJSWI). Tsai (2007) encontrou retornos anormais acumulados negativos na exclusão das ações das empresas, porém nenhuma significância para a inclusão das empresas no índice DJSWI. Por outro lado, Karlsson e Chakarova (2008), mostraram evidencias que ambas as entradas/saídas dos índices de sustentabilidade, em geral, são significativamente anormais para alguns anos e não para outros estudados, concluindo que o conceito de Responsabilidade Social Corporativa (CSR) é tão complexo que tem criado dificuldades para os investidores determinarem o impacto relativo no valor das companhias.

Cheung (2009) mostra num estudo de evento que existem comportamentos anormais nos preços das ações de empresas com ingresso (entrada/saída) no índice de sustentabilidade americano Dow Jones Sustainability Index (DJSWI) para o período de 2002-2007. O estudo analisou a inclusão e 
exclusão de empresas que fizeram parte do índice de acordo com as proxies: retorno, volatilidade e liquidez.

Apesar da diversidade de pesquisas, não há um consenso quanto ao resultado do anúncio (realização) de ingresso (entrada/saída) do Índice de Sustentabilidade. As diferentes metodologias, a composição e período amostral, os indicadores utilizados e as características particulares dos mercados pesquisados são algumas das variáveis influenciadoras destes resultados. Percebe-se um maior interesse pelo comportamento do retorno da ação, amplamente justificado pela sua importância na execução do objetivo maior do acionista; a maximização de sua riqueza. Neste contexto, ressalta-se a utilização dos estudos de eventos como metodologia predominante na análise da reação do retorno das ações (BATISTELA, CORRAR, BERGMANN e AGUIAR, 2004; HACIBEDEL e BOMMEL, 2006; DIAS, 2007 e SALAZAR, 2007).

\section{ASPECTOS METODOLÓGICOS}

\subsection{ESTUDO DE EVENTO}

Para identificar o comportamento do mercado acionário frente ao ingresso (saída) do Índice de Sustentabilidade Empresarial (ISE), a metodologia utilizada é o estudo de evento. Os procedimentos aqui apresentados são realizados para a amostra completa, ou seja, empresas que ingressaram e saíram do ISE tomados em conjunto e, posteriormente, para cada um dos eventos em separado (amostra de entrada e amostra de saída).

Segundo Vachadze (2001), a metodologia de estudo de evento é uma aplicação estatística muito utilizada na área financeira, para estimação no impacto de diferentes divulgações de informações no preço de ações do mercado.

As taxas de retorno observadas de cada empresa $\mathrm{j}$ para o dia t, após um intervalo $i$ decorrido desde a última negociação são calculadas, de acordo com o procedimento "Trade-to-Trade", da seguinte maneira:

$$
R_{j, i t}=\ln \left(\frac{P_{j t}}{P}\right)
$$

onde:

$P j t=$ preço da ação da empresa $j$ no dia $t$;

$i t=$ extensão, no dia $t$, do intervalo de tempo decorrido desde a negociação anterior. Para a identificação da performance anormal, que ocorre se os resíduos forem diferentes de zero, apurou-se os resíduos 
médios ou retornos anormais médios $\left(\mathrm{Ar}_{\mathrm{t}}\right)$ para os períodos $\mathrm{t}$ pertencentes á janela do evento. Sendo o $\mathrm{Ar}_{\mathrm{t}}$ dado pela seguinte equação:

$$
A R_{t}=\frac{1}{N_{j=1}^{N}} \sum_{j t} e
$$

onde:

$\mathrm{N}=$ número de empresas.

Para a identificação da relevância dos resíduos obtidos, além da aplicação de um teste para a significância estatística dos retornos anormais, aplicou-se um Rank Teste conforme proposto por Maynes e Rumsey (1993).

Pela natureza do estudo de evento, a heterocedasticidade dos resíduos apurados exige que os dados utilizados na estimação dos parâmetros da regressão linear sejam divididos pela raiz quadrada do intervalo entre as negociações.

O período utilizado para a estimação dos parâmetros a e b não deve incluir os períodos que compõem o evento. Assim foi utilizado um período de 365 dias corridos, contados a partir do dia anterior a ultima data considerada no período do evento. Como representante do retorno do portfólio de mercado adotou-se o retorno do índice IBOVESPA e o retorno do FGV-100².

A seguir no Quadro 1, apresentam-se as janelas de eventos e os autores de alguns estudos.

Quadro 1 - Janelas de eventos e autores dos estudos

\begin{tabular}{|l|l|l|}
\hline \multicolumn{1}{|c|}{ Período de estimação } & \multicolumn{1}{|c|}{ Janelas de eventos } & \multicolumn{1}{|c|}{ Autores } \\
\hline$-250 \mathrm{a}-30$ dias da data zero & $-5 \mathrm{a}+5$ dias da data zero & Hedge e McDermott (2000) \\
\hline$-31 \mathrm{a}+211$ dias da data zero & $-31 \mathrm{a}+211$ dias da data zero & $\begin{array}{l}\text { Denis, McConnell, Ovtchinnikov e Yu } \\
(2001)\end{array}$ \\
\hline 50 pregões anteriores a -10 dias & $-1 \mathrm{a}+1$ dia da data zero & $\begin{array}{l}\text { Batistella, Corrar, Bergmann e Aguiar } \\
(2004)\end{array}$ \\
\hline$-165 \mathrm{a}-16$ dias da data zero & $-5 \mathrm{a}+5$ dias da data zero & Nast de Lima e Terra (2006) \\
\hline$-10 \mathrm{a}+50$ dias da data zero & $-1 \mathrm{a}+10$ dias da data zero & Hacibedel e Bommel (2006) \\
\hline 5 meses anteriores a -7 dias da & $-1 \mathrm{a}+2$ dias da data zero & Chakarova e Karlsson (2007) \\
\hline data zero & & \\
\hline
\end{tabular}

\footnotetext{
${ }^{2} \mathrm{O}$ objetivo é identificar a interferência do índice de retorno de mercado utilizado nos resultados do estudo de evento.
} 


\begin{tabular}{|l|l|l|}
5 anos anteriores a data zero & $-30 \mathrm{a}+15$ dias da data zero & Salazar (2007) \\
\hline$-15 \mathrm{a}+120$ dias da data zero & $-15 \mathrm{a}+120$ dias da data zero & Dias (2007) \\
\hline 217 pregões anteriores a -10 dias & $-9 \mathrm{a}+9$ dias da data zero & Bogéa, Campos e Camino (2008) \\
\hline $\begin{array}{l}120 \text { pregões anteriores as janela } \\
\text { de }\end{array}$ & $-8 \mathrm{a}+15$ dias da data zero & Luz (2009) \\
\hline $\begin{array}{l}\text { eventos } \\
-250 \mathrm{a}-16 \text { dias da data zero }\end{array}$ & $-15 \mathrm{a}+60$ dias da data zero & Cheung (2009) \\
\hline
\end{tabular}

Como pode ser observado no Quadro 1, não há um consenso sobre a janela ideal e considerando que a aplicação dos dados em painel é desejável que a série temporal de cada ação não seja muito pequena, além da possibilidade da falta de liquidez, o que reduz a quantidade de dados disponíveis. Neste estudo, definiu-se a janela do evento compreendendo um período de 91 dias sendo, 30 dias úteis anteriores (período pré-entrada) e 60 dias úteis posteriores (período pós-entrada) a data “0“. Considerou-se como data "0", a data de entrada em vigor da carteira (01 de dezembro).

\subsection{TESTE PARA A HIPÓTESE DE LIQUIDEZ}

A influência da liquidez sobre os retornos é analisada através da metodologia de análise de dados em painel, pois esta metodologia confronta o padrão de comportamento de uma mesma empresa ao longo do tempo (longitudinalmente) e em relação às outras empresas (cross-section). Segundo Baltagi (2005) esta técnica de análise é uma das mais inovadoras na literatura da econometria, demonstrando um rico ambiente para as técnicas de estimação desenvolvidas e resultados teóricos condizentes.

Hsiao (2003) e Baltagi (2005) listam uma série de benefícios para usar dados em painel, tais como: a) controla a heterogeneidade individual das firmas; b) fornece mais informações; variabilidade e graus de liberdade com menos colinearidade nos dados resultando em maior eficiência; c) é melhor para o ajustamento dinâmico, habilitado para identificar e medir os efeitos que não são fáceis de serem detectáveis nos modelos cross-section e d) permite construir e testar modelos de comportamentos mais complicados do que cross-section. O modelo geral de dados em painel pode ser expresso conforme a fórmula:

$$
y_{i t}=b_{1 i t}+b_{2 i t} x_{1}+\ldots+b_{n i t} x_{n}+e_{i t} .
$$


Onde o subscrito $i=1, \ldots, k$ indica a i-ésima empresa, $t=1, \ldots, T$ indica o tempo e $\beta$ 's são os coeficientes da regressão. As variáveis são dadas por: $y_{i t}=$ variável dependente para a empresa $i$ no tempo $t ; x_{1}=$ variável independente 1 para a empresa $i$ no tempo $t$, sendo neste estudo a reação do mercado; $x_{n}=$ variável independente $n$ para a empresa $i$ no tempo $t ; e_{i t}=$ termo de erro para a empresa $i$ no tempo $t$.

Existem três casos da análise para ajustar modelo geral a fim de torná-lo mais funcional. Modelo Pooled, Fixed-Effects Model (Efeitos Fixos) e o Random Effects (Efeitos Aleatórios). No modelo Pooled o intercepto é o mesmo para toda a amostra, ou seja, assume-se que todos os elementos da amostra possuem comportamento idêntico. No modelo de efeitos fixos, pode haver variação no intercepto entre as empresas e é mais indicado em situações em que o intercepto específico à empresa pode estar correlacionado com um ou mais regressores. No modelo de efeitos aleatórios pressupõe que as empresas sobre as quais se dispõe de dados são uma extração aleatória de uma população com valor médio constante.

Na construção do modelo, primeiramente faz-se necessário verificar se as variáveis possuem associações lineares significativas. Se isto ocorrer, pode-se ter a frente o problema de multicolinearidade que será verificada através do cálculo dos fatores de inflacionamento da variância (VIF), dado por $\operatorname{VIF}(j)=1 /\left(1-\mathrm{R}(j)^{\wedge} 2\right)$, onde $\mathrm{R}(j)$ é o coeficiente de correlação múltipla entre a variável $j$ e as outras variáveis independentes. Se o modelo estiver livre da multicolinearidade, a escolha entre os modelos poderá ser realizada através dos testes específicos.

Como na literatura nacional e internacional, não existe um consenso sobre quais variáveis (proxies) melhor se adéqua um modelo apropriado para analisar liquidez de mercado, neste estudo foram analisadas as seguintes variáveis de liquidez:

- Retorno do índice BOVESPA: retorno do índice benchmark do mercado.

- Volume financeiro: volume financeiro da ação da empresa realizado no dia.

- Volume financeiro ponderado pelo índice IBOVESPA: volume financeiro da ação da empresa dividido pelo volume do IBOVESPA.

- Turnover: quantidade de negócios realizados no dia dividido pela quantidade de ações existentes.

- Relativo bid-ask spread 1 : Preço máximo menos o preço mínimo dividido pela soma dos preços máximo e mínimo dividido por 2 , ou seja, $\{(\max -\operatorname{mim}) /[(\max +\operatorname{mim}) / 2]\}$. 


\subsection{AMOSTRA}

Para este estudo, a amostra é não probabilística, visto que a composição da carteira do Índice ISE é definida pelos critérios do Conselho Deliberativo. $\mathrm{O}$ alvo desta pesquisa são as ações das companhias abertas negociadas na BOVESPA que ingressaram e saíram do ISE no período de 01 de dezembro de 2005 (início da criação da carteira teórica do índice), até 01 de dezembro de 2009 (quinta carteira teórica do índice). Os dados referentes aos preços de fechamento diários das ações no mercado a vista e em moeda original, bem como as demais variáveis foram obtidas através do sistema de informações Economática ${ }^{\circledR}$.

A Tabela 1 demonstra as ações das empresas que entraram e saíram, ano por ano. As saídas são as ações das empresas que deixaram de participar do ISE no índice daquele ano.

Tabela 1 - Quantidade de ações que entraram e saíram do ISE

\begin{tabular}{l|l|c|c}
\hline Ano & Entrada & \multicolumn{1}{c}{ Saída } & Total de ações \\
\hline 2005 & 33 & & 33 \\
2006 & 18 & 6 & 24 \\
2007 & 8 & 11 & 19 \\
2008 & 8 & 10 & 18 \\
2009 & 10 & 5 & 15 \\
Total & 77 & 32 & 109 \\
\hline
\end{tabular}

A Tabela 1 demonstra a quantidade de ações que ingressaram e saíram do ISE, visto que no primeiro ano da carteira teórica do índice, 2005, todas as empresas foram computadas como ingressantes e a partir deste, somente as ações das novas empresas. Foram exclusas da amostra as ações das empresas que não haviam dados completos no sistema de dados do Economática ${ }^{\circledR}$.

O período utilizado para a estimação dos parâmetros $\alpha$ e $\beta$ não deveria incluir os períodos que compõe o evento. Foi utilizado um período de 282 dias úteis, contados a partir do dia anterior à última data considerada no período do evento. Como representantes do retorno de portfólio de mercado foi adotado os índices Bovespa e FGV-100.

\section{RESULTADOS OBTIDOS}

\subsection{ESTUDO DE EVENTO}

Definidos os parâmetros, foram estimados os resíduos diários para uma janela de 91 dias úteis, sendo 30 dias úteis anteriores e 60 dias úteis posteriores à data zero, tanto para o índice de mercado IBOVESPA e FGV 100. A Tabela 2 demonstra os resíduos diários médios apurados para cada uma das amostras, completa, de entrada e saída com os índices IBOVESPA e FGV100. 
Tabela 2 - Resíduos diários médios para a amostra completa, amostra de entrada e amostra de saída com os índices IBOVESPA e FGV100.

\begin{tabular}{|c|c|c|c|c|c|c|c|c|c|c|c|}
\hline \multirow[t]{2}{*}{$\mathrm{t}$} & \multicolumn{4}{|c|}{ Amostra completa } & \multicolumn{4}{|c|}{ Amostra de entrada } & \multicolumn{3}{|c|}{ Amostra de saida } \\
\hline & IBOVES & PA & FGV 10 & & IBOVES & & FGV 10 & & IBOVE & & FGV100 \\
\hline-10 & $-0,09500$ & & $-0,11584$ & & $-0,07396$ & & $-0,12099$ & & $-0,15327$ & & $-0,54476$ \\
\hline-9 & $-0,03342$ & & $-0,03231$ & & 0,00662 & & $-0,03966$ & & $-0,14432$ & & $-1,10216$ \\
\hline-8 & $-0,04404$ & & $-0,07128$ & & $-0,03295$ & & 0,01337 & & $-0,07432$ & & $-0,45483$ \\
\hline-7 & $-0,07027$ & & $-0,05195$ & & $-0,10548$ & & $-0,13247$ & & 0,07058 & & 0,25154 \\
\hline-6 & 0,07192 & & $-0,06231$ & & 0,01026 & & $-0,14254$ & & 0,24267 & & 0,36864 \\
\hline-5 & $-0,05863$ & & 0,04407 & & 0,03878 & & 0,10962 & & $-0,32838$ & & $-0,39434$ \\
\hline-4 & 0,01124 & & 0,00376 & & 0,07526 & & 0,05210 & & $-0,16359$ & & $-0,84919$ \\
\hline-3 & 0,10345 & & 0,03120 & & 0,24057 & 888 & 0,18728 & & $-0,27626$ & & $-1,91410$ \\
\hline-2 & 0,04183 & & 0,05489 & & $-0,04619$ & & 0,00313 & & 0,28219 & & 1,04678 \\
\hline-1 & 0,14958 & **** & 0,13590 & 88 & 0,17187 & ** & 0,28830 & ** & 0,08955 & & 0,04337 \\
\hline 0 & $-0,12774$ & & $-0,06670$ & & $-0,03535$ & & 0,01562 & & $-0,38358$ & **** & $-1,39869$ \\
\hline 1 & $-0,11341$ & $* * *$ & $-0,14630$ & $\$ * 8$ & $-0,12801$ & & $-0,21560$ & $* * *$ & $-0,07296$ & & $-0,05482$ \\
\hline 2 & 0,06138 & & 0,07576 & & $-0,04287$ & & $-0,10967$ & & 0,34608 & **** & 2,46196 \\
\hline 3 & 0,08845 & & 0,12598 & & 0,19599 & $\$ 8 \%$ & 0,30793 & ** & $-0,20936$ & & $-1,10927$ \\
\hline 4 & $-0,03893$ & & $-0,06841$ & & $-0,09750$ & & $-0,07801$ & & 0,12326 & & 0,66744 \\
\hline 5 & $-0,13127$ & & $-0,25298$ & & $-0,09141$ & & $-0,12560$ & & $-0,24012$ & & $-1,75776$ \\
\hline 6 & $-0,31617$ & * & $-0,32977$ & $*$ & $-0,32674$ & $*$ & $-0,42369$ & * & $-0,28691$ & & $-1,04400$ \\
\hline 7 & $-0,16971$ & & $-0,05536$ & & $-0,15592$ & & $-0,09473$ & & $-0,20787$ & & 0,21516 \\
\hline 8 & 0,13022 & & 0,10873 & & 0,20898 & & 0,09114 & & $-0,08790$ & & $-0,35605$ \\
\hline 9 & $-0,13535$ & $* * *$ & $-0,21206$ & 88 & $-0,09282$ & & $-0,19905$ & $* * *$ & $-0,25614$ & & $-1,54379^{* * *}$ \\
\hline 10 & $-0,03049$ & & $-0,09171$ & & $-0,01452$ & & 0,00605 & & $-0,07347$ & & $-0,81073$ \\
\hline
\end{tabular}

*significativo ao nível de $1 \%$

**significativo ao nível de $5 \%$

***significativo ao nível de $10 \%$

Verifica-se na Tabela 2 que na amostra completa, ocorreram alterações negativas significativas, em $\mathrm{t}_{1}(-0,11 \%), \mathrm{t}_{6}(-0,31 \%)$ e em $\mathrm{t}_{9}(-0,13 \%)$ e uma única alteração positiva, com significância de $10 \%$, em t-1 $(0,14 \%)$. Já para a amostra de entrada, a única alteração negativa, com significância de $1 \%$, é verificada no instante $t_{6}(-0,32 \%)$, sendo que este instante apresenta a maior alteração negativa nesta amostra e as alterações positivas significativas para a amostra de entrada são: em $\mathrm{t}_{-3}(0,24 \%), \mathrm{t}_{-1}(0,17 \%)$ e em $\mathrm{t}_{3}(0,19 \%)$, podendo ser uma evidencia de informação positiva do mercado acerca da entrada das ações no índice ISE. Já para a amostra de saída ocorreu alteração positiva, com significância de $10 \%$ em $t_{2}(0,35 \%)$ e alteração negativa em $\mathrm{t}_{0}(-0,38 \%)$. Este instante $\mathrm{t}$ 0 representa a maior alteração desta amostra e também o maior valor médio percentual de toda a Tabela 2. Isto pode ser uma evidência de que os investidores reagem negativamente à saída das ações do ISE. Para a amostra de entrada, o resíduo não é significativo, portanto, na data do evento, em termos médios, o mercado reage significativamente apenas para as saídas de índice. Este resultado é coerente com os resultados obtidos por Cheung, 2009, que verificou diminuição nos retornos das ações após serem excluídas do índice de sustentabilidade DJSWI (Dow Jones Sustainability World Index) durante o período 2002-2007. Por outro lado o trabalho de Moura, 2009, evidenciou nenhum comportamento no rendimento de ações que foram inclusas (exclusas) do índice BOVESPA, no período 1990-2008. Da mesma forma o estudo de Dias, 2007, não encontrou retornos anormais em datas próximas ao anúncio da inclusão (exclusão), das ações das empresas que fizeram parte do ISE para os anos 2006-2007. 


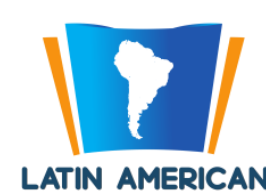

LATIN AMERICAN

Para análise dos resíduos diários médios das amostras com o índice FGV 100, apresentam valores significativos negativos na amostra completa em $\mathrm{t}_{-1}(-0,14 \%), \mathrm{t}_{6}(-0,32 \%)$ e em $\mathrm{t}_{9}(-0,21 \%)$, com valor positivo, com significância de 5\%, em t-1 (0,13\%). Já na amostra de entrada, os valores positivos significativos, são demonstrados nos instantes t-1 $(0,29 \%)$ e $t_{3}(0,31 \%)$, os significativamente negativos são respectivamente nos instantes: $t_{1}(-0,21 \%), t_{6}(-0,42 \%)$ e em $t_{9}(-0,19 \%)$. Porém, na amostra de saída, tem-se um único valor negativo significativo, em t9 (-1,54\%), sendo o maior valor residual desta amostra e da Tabela 2. Comparativamente aos resultados obtidos com o índice BOVESPA, observa-se que com o índice FGV-100, a amostra de saída passou a não ser significativa na data zero, indicando não haver retornos anormais. Nos demais períodos não há grandes diferenças entre os dois resultados, pois a maioria dos coeficientes dos retornos não são significativos. As Figuras 1 e 2 podem demonstrar melhor o comportamento dos resíduos diários médios para cada uma das amostras com os índices IBOVESPA e FGV 100.

Figura 1 - Resíduos diários médios da amostra completa, amostra de entrada e amostra de saída com o índice BOVESPA

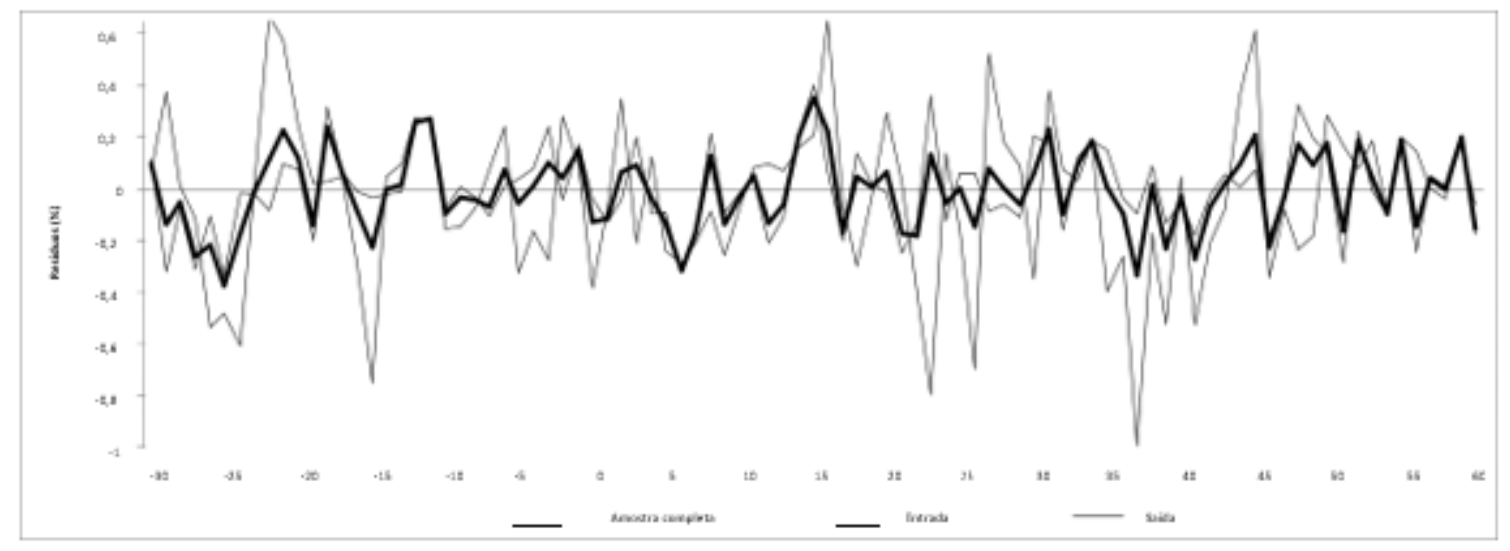

Figura 2 - Resíduos diários médios da amostra completa, amostra de entrada e amostra de saída com o índice FGV 100

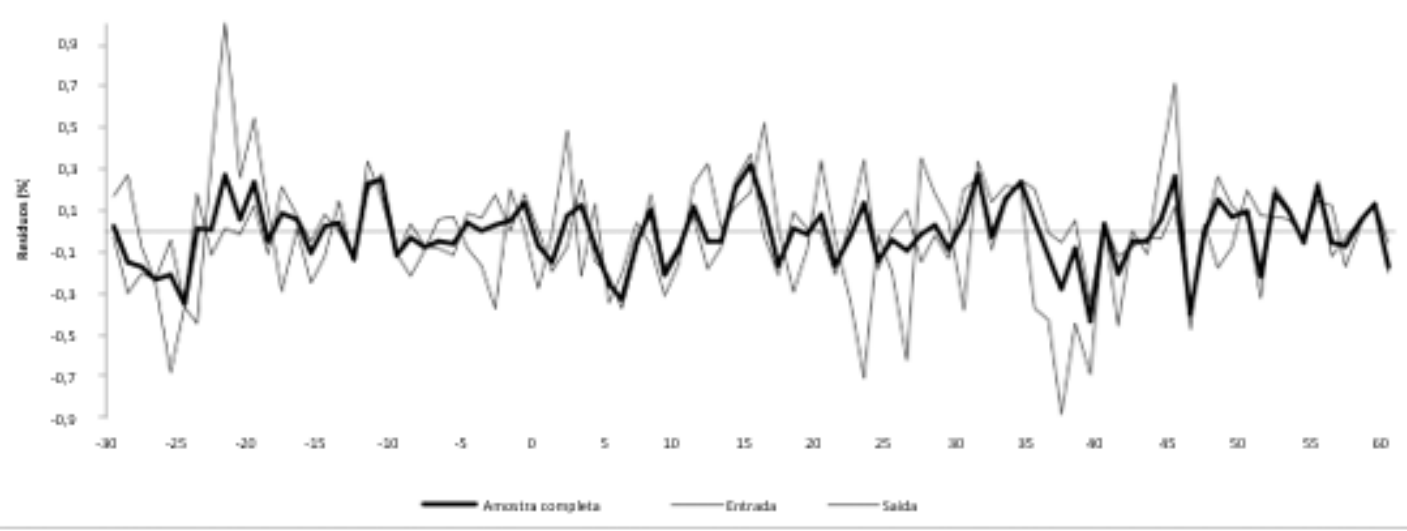


As Figuras 1 e 2, apresentam comportamentos semelhantes ao longo da janela do evento, indicando que, em termos gerais, o índice de mercado utilizado não promove grandes alterações nos resultados. No entanto três pontos merecem destaque. Primeiro, a amostra de saída, nos dois casos (BOVESPA e FGV 100) são os que apresentam as maiores oscilações dentre as três amostras, o que indica uma maior variação dos retornos anormais. Segundo, nos dias mais próximos à data do evento, as oscilações dos retornos anormais, nas três amostras, são menores. Terceiro, nos dias próximos à data do evento $(-5 \mathrm{a}+5)$, a maioria dos retornos anormais da amostra de entrada apresenta sinal contrário ao da amostra de saída.

Para investigar uma possível tendência ao longo do período, optou-se por acumular os resíduos diários em intervalos e para toda a janela do evento. Os resultados se encontram na Tabela 3.

Tabela 3 - Resíduos acumulados para a amostra completa, de entrada e saída com os índices BOVESPA e FGV 100

\begin{tabular}{|c|c|c|c|c|c|c|c|c|c|c|c|c|c|c|c|c|c|c|}
\hline \multirow[b]{3}{*}{$\mathrm{t}$} & \multicolumn{3}{|c|}{ IBOVESPA } & \multicolumn{3}{|c|}{$\begin{array}{l}\text { FGV } \\
100\end{array}$} & \multicolumn{3}{|c|}{ IBOVESPA } & \multicolumn{3}{|c|}{$\begin{array}{l}\text { FGV } \\
100\end{array}$} & \multicolumn{3}{|c|}{ IBOVESPA } & \multicolumn{3}{|c|}{$\begin{array}{l}\text { FGV } \\
100\end{array}$} \\
\hline & \multicolumn{6}{|c|}{ Amostra completa } & \multicolumn{6}{|c|}{ Amostra entrada } & \multicolumn{6}{|c|}{$\begin{array}{l}\text { Amostra } \\
\text { saída }\end{array}$} \\
\hline & $(\%)$ & $Z$ & & $(\%)$ & $Z$ & & $(\%)$ & $Z$ & & $(\%)$ & $Z$ & & $(\%)$ & $Z$ & & $(\%)$ & $\mathrm{Z}$ & \\
\hline$-30 a-25$ & 0,37 & 3,69 & $*$ & 0,35 & 3,50 & $*$ & 0,33 & 2,84 & $*$ & 0,33 & 2,95 & $*$ & 0,48 & 2,45 & $* *$ & 0,18 & 1,87 & $* * *$ \\
\hline-24 a -20 & 0,12 & 1,18 & $* *$ & 0,24 & 2,33 & $* *$ & 0,07 & 0,61 & $*$ & 0,09 & 1,04 & & 0,25 & 1,26 & & 0,44 & 2,80 & $*$ \\
\hline$-19 a-15$ & 0,23 & 2,22 & $* *$ & 0,03 & 0,26 & & 0,03 & 0,28 & & 0,10 & 0,68 & & 0,75 & 3,82 & $*$ & 0,18 & 0,61 & \\
\hline$-14 \mathrm{a}-10$ & 0,10 & 0,94 & & 0,12 & 1,15 & & 0,07 & 0,63 & & 0,12 & 1,01 & & 0,15 & 0,78 & & 0,23 & 0,54 & \\
\hline$-9 a-5$ & 0,06 & 0,58 & & 0,04 & 0,44 & & 0,04 & 0,33 & & 0,11 & 0,75 & & 0,33 & 1,67 & & 0,23 & 0,39 & \\
\hline-4 a 0 & 0,13 & 1,26 & & 0,07 & 0,66 & & 0,04 & 0,30 & & 0,02 & 0,07 & & 0,38 & 1,96 & $* * *$ & 0,24 & 1,40 & \\
\hline 1 a 5 & 0,13 & 1,29 & $* * *$ & 0,25 & 2,49 & $* *$ & 0,09 & 0,77 & & 0,13 & 1,85 & $* * *$ & 0,24 & 1,22 & & 0,19 & 1,76 & $* * *$ \\
\hline 6 a 10 & 0,03 & 0,30 & $*$ & 0,09 & 0,90 & & 0,01 & 0,12 & & 0,01 & 0,56 & & 0,07 & 0,37 & & 0,11 & 0,81 & \\
\hline 11 a 15 & 0,35 & 3,45 & $*$ & 0,32 & 3,19 & $*$ & 0,40 & $\beta, 40$ & $*$ & 0,25 & 3,14 & $*$ & 0,21 & 1,05 & & 0,10 & 0,97 & \\
\hline 16 a 20 & 0,06 & 0,59 & $*$ & 0,08 & 0,79 & & 0,01 & 0,11 & $*$ & 0,04 & 0,03 & & 0,29 & 1,40 & & 0,34 & 1,64 & \\
\hline 21 a 25 & 0,00 & 0,04 & & 0,04 & 0,42 & & 0,06 & 0,51 & & 0,02 & 0,08 & & 0,16 & 0,80 & & 0,15 & 0,97 & \\
\hline 26 a 30 & 0,06 & 0,59 & & 0,05 & 0,52 & & 0,20 & 1,70 & $* * *$ & 0,16 & 1,72 & $* * *$ & 0,35 & 1,74 & $* * *$ & 0,37 & 1,89 & $* * *$ \\
\hline 31 a 35 & 0,01 & 0,09 & $* *$ & 0,05 & 0,54 & & 0,15 & 1,28 & & 0,13 & 1,73 & $* * *$ & 0,39 & 1,97 & $* * *$ & 0,24 & 1,87 & $* * *$ \\
\hline 36 a 40 & 0,03 & 0,27 & $* *$ & 0,04 & 0,37 & & 0,05 & 0,45 & & 0,02 & 0,31 & & 0,05 & 0,24 & & 0,06 & 0,21 & \\
\hline 41 a 45 & 0,21 & 2,03 & $* *$ & 0,27 & 2,62 & $* *$ & 0,07 & 0,60 & & 0,07 & 1,01 & & 0,61 & 2,99 & $*$ & 0,49 & 3,48 & $*$ \\
\hline 46 a 50 & 0,18 & 1,74 & $* * *$ & 0,10 & 1,01 & & 0,14 & 1,17 & & 0,09 & 0,59 & & 0,29 & 1,43 & & 0,17 & 1,00 & \\
\hline 51 a 55 & 0,19 & 1,87 & $* * *$ & 0,19 & 1,87 & $* * *$ & 0,19 & 1,59 & & 0,23 & 2,08 & $* *$ & 0,20 & 0,99 & & 0,13 & 0,73 & \\
\hline 56 a 60 & 0,13 & 0,20 & & 0,22 & 2,17 & $* *$ & 0,03 & 0,28 & & 0,09 & 0,43 & & 0,43 & 0,10 & & 0,16 & 0,05 & \\
\hline 0 a 5 & 0,13 & 0,04 & & 0,08 & 0,08 & & 0,05 & 0,10 & & 0,08 & 0,15 & & 0,20 & 0,09 & & 0,42 & 0,10 & \\
\hline-30 a 60 & 0,74 & 0,13 & & 0,97 & 0,15 & & 0,11 & 0,14 & & 1,23 & 0,16 & & 3,38 & 0,02 & & 6,63 & 0,02 & \\
\hline
\end{tabular}

*significativo ao nível de $1 \%$

**significativo ao nível de $5 \%$

***significativo ao nível de $10 \%$

Observa-se na Tabela 3 que no intervalo total (-30 a 60), bem como no intervalo em torno da janela do evento ( 0 a 5), não foram obtidos retornos acumulados com significância, portanto em termos gerais estes intervalos não são estatisticamente significativos. Porém vale salientar que dentro das amostras existem valores com coeficientes com significância de 1, 5 e 10\%, próximos a data do evento, 
na amostra completa, nos intervalos $\mathrm{t}_{1}$ até $\mathrm{t}_{20}(-013 \%),(-0,03 \%),(0,34 \%)$ e $(0,06 \%)$ respectivamente. Já para a amostra de saída, no intervalo t-4 a to (- 0,38\%), com significância de $10 \%$. Anteriores a data do evento, são verificados os intervalos significativos em t-30 até t $-15(-0,37 \%),(-0,11 \%)$ e $(-0,22 \%)$ respectivamente para a amostra completa, os intervalos t-30 até t-20 $(-0,33 \%)$ e $(0,07 \%)$ para a amostra de entrada e os intervalos $\mathrm{t}_{-30}$ a t-25 (-0,48\%) e $\mathrm{t}_{-19}$ a t-15 (-0,74\%) na amostra de saída com o índice BOVESPA. Posteriores a data do evento, encontram-se coeficientes significativos em $t_{31}$ até t55 $(0,009 \%),(-0,27 \%),(0,20 \%),(0,17 \%)$ e $(0,19 \%)$ na amostra completa, na amostra de entrada, não existem valores significativos próximos a data do evento, mas podemos destacar intervalo $t_{11}$ a $t_{15}$ $(0,40 \%)$ maior valor residual desta amostra, $\mathrm{t}_{16}$ a $\mathrm{t}_{20}(-0,01 \%)$, ambos significativos a $1 \%$ e o intervalo $\mathrm{t}_{26}$ a $\mathrm{t}_{30}(0,20 \%)$, significativo a $10 \%$. Já para a amostra de saída, têm-se os intervalos $\mathrm{t}_{26}$ a $\mathrm{t}_{30}(-0,34 \%)$ e $t_{31}$ a $t_{35}(-0,39 \%)$, negativos e significativos, apenas o intervalo $t_{41}$ a $t_{45}(0,61 \%)$ aparece com significância de $1 \%$ e positivo.

Já para os resíduos acumulados das amostras completa, de entrada e saída com o índice FGV 100, identificam-se coeficientes significativos próximos a data zero, no intervalo $t_{1}$ a $t_{5}$, para ambas as amostras. Significativo a $5 \%(-0,25 \%)$ na amostra completa, significativos a $10 \%(-0,12 \%)$ na amostra de entrada e significativos a $10 \%(-0,19 \%)$ na amostra de saída. Anterior a data zero, tem-se coeficientes significativos apenas em $\mathrm{t}_{-30}$ até $\mathrm{t}_{-20},(-0,35 \%)$ e $(0,23 \%)$ para a amostra completa, o

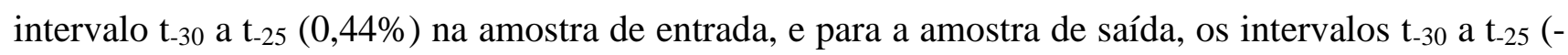
$035 \%)$ e t-24 a t-20 (-0,32\%). Posteriores a data do evento observa-se na amostra completa, os intervalos $\mathrm{t}_{41}$ a $\mathrm{t}_{45}(0,26 \%)$ e $\mathrm{t}_{51}$ até $\mathrm{t}_{60}(0,19 \%)$ e $(0,22 \%)$ respectivamente. Já para a amostra de entrada, têm-se os intervalos $\mathrm{t}_{11}$ a $\mathrm{t}_{15}(0,24 \%), \mathrm{t}_{26}$ até $\mathrm{t}_{35}(0,16 \%)$ e $(0,12 \%)$ respectivamente, e o intervalo $\mathrm{t}_{51}$ a $\mathrm{t}_{55}$ $(0,23 \%)$ com significância de $1 \%$. Por última análise da Tabela 3, na amostra de saída, posteriores a data zero, encontram-se os intervalos com significância em $\mathrm{t}_{26}$ até $\mathrm{t}_{40}(-0,36 \%)$ e $(-0,24 \%)$, e em $\mathrm{t}_{41}$ a $\mathrm{t}_{45}(0,48 \%)$.

Assim pode-se verificar um comportamento distinto em torno da data do evento, antes e depois da entrada e saída do índice ISE na Tabela 3. Na amostra de entrada com o índice BOVESPA, verificam-se dois coeficientes positivos frente a um coeficiente negativo. Após a entrada do índice ISE e na amostra de saída, tem-se três coeficientes negativos (um anterior e dois posteriores a data do evento) em relação a um coeficiente positivo. Isto pode ser uma evidencia de que o mercado reage negativamente à saída do índice ISE e positivamente à sua entrada no referido índice. Os resíduos acumulados das amostras com o índice FGV 100 são verificados coeficientes negativos anteriores a data do evento, para ambas as amostras. Já na amostra de entrada, após a data do evento, têm-se apenas coeficientes positivos, com significância de 1, 5 e 10\%. Porém, na amostra de saída, após a data do evento, verificam-se dois coeficientes negativos, com significância de 10\%, em relação a um 
coeficiente positivo, significativo a $1 \%$. Portanto, a análise com relação à entrada das ações das empresas que aderem ao índice ISE, assim como à saída das ações das empresas do referido índice, há evidência de que o mercado reage positivamente a sua entrada e negativamente a sua saída. Porém, como metades das ações que participam do índice ISE, participam também do índice BOVESPA, podem os resultados ser influenciados pelos índices de mercado utilizados na amostra, estes derivados das diferenças amostrais, no índice FGV 100, pelo qual as instituições financeiras não fazem parte da carteira teórica deste índice e estas integram em parte a carteira teórica do índice BOVESPA.

\subsection{TESTE DE LIQUIDEZ}

Nesta fase da pesquisa, foram analisadas as amostras completa, de entrada e saída da carteira teórica do ISE para os testes de liquidez. Como no estudo de evento realizaram-se os cálculos para estimação dos betas e apuraram-se os retornos diários para cada uma das ações das empresas no intervalo -30 a +60, com os índices BOVESPA e FGV 100, os parâmetros de liquidez foram definidos as seguintes variáveis independentes: retorno do índice BOVESPA, volume financeiro, volume financeiro ponderado pelo índice BOVESPA, turnover e relativo bid-ask spread.

Inicialmente foram calculados os valores de cada uma das variáveis para cada uma das amostras, no período compreendido entre os 30 dias anteriores à data zero e os 60 dias posteriores. Portanto a amostra completa é formada por 99 ações, a amostra de entrada, formada por 72 ações e a amostra de saída formada com 27 ações das empresas que compuseram as entradas e as saídas do índice ISE para os anos 2005-2009. Todos os dados na série temporal em torno do evento compreendem o mesmo período $(-30$ a +60$)$. A amostra completa considera as duas séries anteriores indistintamente por terem sido consideradas apenas os dias com negociação, portanto, trata-se de um painel desbalanceado, já que o tamanho da série temporal de cada ação depende de quantos dias ao longo do período $(-30 \mathrm{a}+60)$ houve negociação.

Nas Tabelas 4 e 5, foram calculadas as médias, os desvios padrão e verificadas as significâncias para o retorno das empresas (como variável dependente), com o retorno do índice BOVESPA, o retorno do índice FGV 100 e as variáveis de liquidez.

Tabela 4 - Médias dos retornos das ações das empresas na amostra completa, de entrada e de saída com o índice BOVESPA

\begin{tabular}{|c|c|c|c|c|c|c|c|c|c|}
\hline \multirow[t]{2}{*}{ Variável } & \multicolumn{3}{|c|}{$\begin{array}{l}\text { Amostra } \\
\text { completa }\end{array}$} & \multicolumn{3}{|c|}{ Amostra entrada } & \multicolumn{3}{|c|}{$\begin{array}{l}\text { Amostra } \\
\text { saída }\end{array}$} \\
\hline & Coeficiente & E.P. & Sig. & Coeficiente & E.P. & Sig. & Coeficiente & E.P. & Sig. \\
\hline Retorno IBOV & 0,8241208 & 0,0147204 & 0,000 & 0,7734585 & 0,17033 & 0,000 & 0,7392591 & 0,0362287 & 0,000 \\
\hline Turnover & 1,42285 & 0,1372211 & 0,000 & 0,0869778 & 0,1336862 & 0,515 & 0,1171493 & 0,1570498 & 0,456 \\
\hline $\begin{array}{l}\text { Volume } \\
\text { Volpond }\end{array}$ & $1,78 \mathrm{e}-13$ & $2,10 \mathrm{e}-12$ & 0,933 & $9,73 e-13$ & $1,62 \mathrm{e}-12$ & 0,633 & $-5,96 \mathrm{e}-12$ & $1,20 \mathrm{e}-11$ & 0,618 \\
\hline IBOV & $-0,0178314$ & 0,0167417 & 0,287 & 0,0064495 & 0,0181397 & 0,722 & 0,0204991 & 0,431615 & 0,635 \\
\hline
\end{tabular}


Analisando a amostra completa na Tabela 4, pode-se perceber que, em média existe retorno positivo com o índice BOVESPA, com o turnover e com o relativo spread1, as demais variáveis não apresentaram significância. Já para a amostra de entrada e amostra de saída, a única variável que apresentou, em média, coeficiente significativo, foi a variável retorno do índice BOVESPA.

A seguir o modelo regressivo passou a ser analisado com o índice FGV 100. Da mesma forma, a análise procedeu-se como verificado com o índice BOVESPA. A Tabela 5 analisa as médias dos retornos das ações das empresas com o retorno do índice FGV 100, para as amostras completa, de entrada e de saída do índice ISE.

Tabela 5 - Médias dos retornos das ações das empresas na amostra completa, de entrada e saída com o índice FGV 100

\begin{tabular}{|c|c|c|c|c|c|c|c|c|c|}
\hline \multirow[t]{2}{*}{ Variável } & \multicolumn{3}{|c|}{$\begin{array}{l}\text { Amostra } \\
\text { completa }\end{array}$} & \multicolumn{3}{|c|}{$\begin{array}{l}\text { Amostra } \\
\text { entrada }\end{array}$} & \multicolumn{3}{|c|}{$\begin{array}{l}\text { Amostra } \\
\text { saída }\end{array}$} \\
\hline & Coeficiente & E.P. & Sig. & Coeficiente & E.P. & Sig. & Coeficiente & E.P. & Sig. \\
\hline Retorno FGV & 0,8506467 & 0,0170645 & 0,000 & 0,8840505 & 0,020161 & 0,000 & 0,9320409 & 0,0469246 & 0,000 \\
\hline Turnover & 1,092725 & 0,1706093 & 0,000 & 0,1093207 & 0,1347236 & 0,417 & 0,1094219 & 0,1577102 & 0,488 \\
\hline Volume & $-4,09 e-13$ & $2,93 \mathrm{e}-12$ & 0,889 & $7,86 e-13$ & $1,63 \mathrm{e}-12$ & 0,630 & $-6,68 \mathrm{e}-12$ & $1,20 \mathrm{e}-11$ & 0,578 \\
\hline Vol & & & & & & & & & \\
\hline IBOV & $-0,0168333$ & 0,024115 & 0,485 & 0,0058658 & 0,0182796 & 0,748 & 0,0231252 & 0,0433448 & 0,594 \\
\hline Spread1 & $-0,0472161$ & 0,0240368 & 0,049 & $-0,0165987$ & 0,0246352 & 0,500 & 0,119021 & 0,277084 & 0,668 \\
\hline
\end{tabular}

Analisando a Tabela 5, com a variável independente o índice FGV 100 e as variáveis de liquidez, novamente as variáveis com significância na amostra completa, foram o retorno do índice, o turnover e o relativo spread1. Nas demais amostras, de entrada e de saída, não houveram variáveis com coeficientes, em média, diferentes de zero, salvo o retorno com o índice FGV 100, com significância em ambas amostras.

Em conjunto, estes resultados permitem definir que somente na amostra completa temos variáveis explicativas quanto à liquidez, com dados significativos e positivos para o retorno dos índices, BOVESPA e FGV 100, turnover e relativo spread1. No restante das amostras, de entrada e de saída, não houve alterações com dados significativos para as variáveis de liquidez, apenas para o retorno com os índices, BOVESPA e FGV 100.

\section{CONCLUSÕES}

O presente trabalho procurou avaliar a reação dos investidores quanto à entrada e a saída das ações das empresas que aderem ao índice de Sustentabilidade Empresarial (ISE) da Bolsa de Valores de São Paulo para os anos de 2005-2009, e identificar possíveis alterações nos níveis de liquidez de algumas variáveis. 
Apesar de ter encontrado comportamento anormal dos preços das ações com resíduos positivos encontrados na amostra de entrada com o índice BOVESPA, indicando que as taxas de retornos observadas das empresas que entraram no índice ISE, foram superiores às taxas de retorno esperadas, não se pode ter alguma evidência de anormalidade nos resíduos dos preços das ações das empresas, visto que em todas as amostras são verificados resíduos positivos seguidos de negativos e vice-versa. A mesma análise pode ser verificada na amostra de saída do índice ISE com o índice BOVESPA, no instante $t_{0}$ (data do evento), é verificado o maior valor residual negativo neste instante, $-0,38 \%$. Já para análise com o índice FGV 100, são verificados instantes anteriores e posteriores a data zero com valores residuais positivos para a amostra de entrada ao índice ISE e na amostra de saída, foi verificado um único resíduo negativo. Portanto, esta análise pode ser uma evidência de que os investidores estariam reagindo positivamente à entrada das ações das empresas ao ISE, ao mesmo tempo em que estariam reagindo negativamente à saída das ações no mesmo índice. Esta evidência é marcada nas amostras de entrada, com os índices BOVESPA e FGV 100, com resíduos positivos próximos à data do evento, evidenciando que os investidores estariam se antecipando aos resultados ou devido a um vazamento de informações. Ações excluídas do índice tiveram uma diferença maior do que as ações incluídas em todas as amostras analisadas.

Para os testes de liquidez, houve relação positiva na amostra completa, com o retorno das empresas e as variáveis: retorno dos índices, BOVESPA E FGV 100 e turnover. Relação negativa somente foi verificada com a variável relativo spread1. Porém, para a amostra de entrada (ingresso) e amostra de saída (exclusão) das ações da carteira teórica do ISE, houve relação positiva somente entre o retorno das ações das empresas com o retorno dos índices BOVESPA e FGV 100. Isto, talvez possa ser explicado pela limitação deste trabalho, visto que o fato de a ação sair ou entrar no ISE, pode não ter o efeito esperado em termos de alteração na liquidez, uma vez que a efetiva participação das ações participantes no ISE são também ações participantes do BOVESPA, índice amplamente utilizado como benchmark do mercado.

Porém, confrontando os resultados obtidos para todas as amostras, não há evidencia de retornos anormais, ou seja, de que a interpretação dos investidores à entrada e saída do índice ISE, seja favorável à possibilidade de realização de retornos acima dos esperados, pois há evidencia de resíduos positivos seguido de resíduos negativos dentro de uma mesma amostra, considerando assim, que sua reação quanto à participação das ações das empresas no índice ISE, seja somente, parte da obrigação das empresas com reconhecimento em práticas socialmente responsáveis e desenvolvimento sustentável ganhando com isto - apenas um selo de qualidade reconhecida como sustentável no longo prazo.

Desta forma, pode-se ainda inferir que os resultados estão de acordo com a Eficiência de Mercado (FAMA, 1970). Segundo a teoria de eficiência de mercado na forma semi-forte, as práticas 
das empresas que fazem parte do Índice de Sustentabilidade Empresarial já estão presentes nos preços das ações, portanto, em datas anteriores ou posteriores ao anuncio da entrada ou saída da carteira teórica do índice, não existem anormalidades nos retornos das ações das empresas.

No entanto, cabe destacar que apesar deste trabalho não ter encontrado evidências de retornos extras em termos da performance das ações, corrobora com os trabalhos de COSTA, 2007, DIAS, 2007, e BOGÉA, SABER e CAMINO, 2008. Assim, poderão existir outros ganhos em termos de performance, como por exemplo, em termos de demonstrativos contábeis, índices de retorno e lucratividade. Do mesmo modo, pode haver melhorias na empresa em diversos outros temas não estudados aqui neste trabalho, como por exemplo, na imagem da empresa perante seus consumidores.

Finalmente, surge a necessidade de se realizarem novos estudos sobre o ingresso (saída) das ações das empresas ao Índice de Sustentabilidade Empresarial, a fim de identificar o comportamento do mercado acionário com as práticas sustentáveis das empresas. A associação entre os resultados do estudo de evento e testes com variáveis de liquidez, através de um modelo estatístico adequado, poderá, futuramente, indicar um melhor modelo explicativo, trazendo retorno para os acionistas e para sociedade como um todo. 


\section{REFERÊNCIAS}

ARANTES, E. Investimento em responsabilidade social e sua relação com o desempenho econômico das empresas. Conhecimento interativo, São José dos Pinhais, PR, v. 2, n. 1, p. 03-09, jan./jun. 2006.

BATISTELLA, F.D.; CORRAR, L.Z.; BERGMANN, D.R.; AGUIAR, A. D. Retorno de ações e governança corporativa: um estudo de eventos. IV Congresso de Controladoria e contabilidade, USP. São Paulo. 2004

BECCHETTI, L.; DI GIACOMO, S.; PINNACCHIO, D. Corporate social responsibility and corporate performance: Evidence from a panel of US listed companies. Research Paper Series, v. 26, n. 78, dez/2005. Disponível em <www.ssrn.com>. Acesso em 30 de agosto de 2009.

BOGÉA, F.; CAMPOS, A.S.; CAMINO, D. Did the creation of ISE created value to companies. XXXII Encontro da Anpad, Rio de Janeiro. 2008.

BORBA, Paulo R. T. Relação entre desempenho social corporativo e desempenho financeiro de empresas no Brasil. 2005. Dissertação de Mestrado. Disponível em <www.furb.com.br>. Acesso em 03 de março de 2010.

BOVESPA. Bolsa de Valores de São Paulo. São Paulo, 2008. Disponível em <http://www.bmfbovespa.com.br/indices/BuscarIndices.aspx?idioma=pt-br>. Acesso em 28 de dezembro de 2009.

_. Sustentabilidade na BM\&FBOVESPA. São Paulo, 2009. Disponível em

$<$ http://www.bmfbovespa.com.br/pt-br/a-

bmfbovespa/sustentabilidade/sustentabilidade.aspx?idioma=pt-br $>$. Acesso em 04 de janeiro

de 2010.

CEZAR, Jesuina F.; JÚNIOR, Annor da Silva . A relação entre responsabilidade social e ambiental com o desempenho financeiro: Um estudo empírico na Bovespa no Período de 1999 a 2006. In: ANPCONT, 2008, Salvador. II ANPCONT - Os novos paradigmas das Ciências Contábeis, 2008.

CHEUNG, A.W.K. Do stock investors value corporate sustainability? Evidence form an event study. 2009. Disponível em <www.ssrn.com>. Acesso em 05 de novembro de 2009. COSTA, F.J.M. Sustentabilidade e desempenho financeiro: uma análise do mercado brasileiro de ações, 2007. 105 p. (Mestrado Profissional em Administração de Empresas) Núcleo de Pós-Graduação em Administração da Escola de Administração da Universidade Federal da Bahia. Bahia.

DENIS, D.K; MCCONNELL, J.J.; OVTCHINNIKOV, A.V.; YU, Y. $\quad$ S\&P 500 Index additions and earnings expectations. 200.1 Disponível em <

http://papers.ssrn.com/sol3/papers.cfm?abstract_id=329543>. Acesso em 30 de março de 2010.

DIAS, Edson A. Índice de Sustentabilidade Empresarial e retorno ao acionista: Um $\begin{array}{lllll}\text { estudo de } & \text { evento. } & \text { Disponível } & \text { em }\end{array}$

<http://mx.mackenzie.com.br/tede/tde_busca/arquivo.php?codArquivo=697>. Acesso em 25 de novembro de 2009. 
FAMA, Eugene F.; FISHER, Lawrence; JENSEN, Michael \& ROLL, Richard. The adjustment of stock prices to new information. International Economic Review, v. 10 n. 1, p. 1-21, 1970.

FAMA, Eugene F. Efficient capital markets: II. Journal of Finance, v.46, n.5, p. 1575-1617, 1991.

FINCH, N. The emergence of CSR and sustainability indices. 2006. Disponível em <www.ssrn.com>. Acesso em 16 de março de 2010.

HACIDEBEL, B.; BOMMEL, J.V. Do emerging market stocks benefit from index inclusion? 2006. Disponível em <http://econpapers.repec.org/paper/mmfmmfc06/128.htm>. Acesso em 31 de março de 2010.

HILEMAN, D.; BESLY, M.J.; SAVITZ, A. Enviromental quality management. Willey Periodicals, Inc. v.16, issue 1, p.25-46, 2006.

KARLSSON, J., CHAKAROVA, Y. Does corporate social responsibility pay off? An event study of the impact of corporate entry and exit from the Dow Jones Sustainability World Index on the market value of a company. 2008. Disponível em <www.essay.se/ essay/cc87784398/>. Acesso em 26 de novembro de 2009.

LO S. e SHEU, H. Is Corporate sustainability a value-increasing strategy for business? corporate governance: An International Review, v.15, n.2, p.345-358. 2007.

LÓPEZ, M.V.; GARCIA, A.; RODRIGUEZ, L. Sustainable development and corporate performance: A study based on the Dow Jones Sustainability Index. Journal of Business Ethics, 75:285-300, 2007.

MACKEY, A.; MACKEY, T.; BARNEY, J. Corporate social responsibility and firm performance: investor preference and corporate strategies. Fourthcoming in Academy of Management Review. 2005. Disponível em <www.ssrn.com>. Acesso em 01 de março de 2010.

MAKNI, R.; FRANCOEUR, C.; BELLAVANCE, F. Causality between corporate social performance and financial performance: evidence from Canadian firms. Journal of Business Ethics. Springer 2008. Disponível em <www.ssrn.com>. Acesso em 22 de janeiro de 2010. MAYNES, Elizabeth \& RUMSEY, John. Conducting event studies with thinly traded stocks.

Journal of Banking and Finance, v. 17, p. 145-157, 1993.

MEADOWS, D.; RANDERS, J.; MEADOWS, D. Limites do crescimento: a atualização de

30 anos. Rio de Janeiro: Qualitymark, 2007.

MEDEIROS, O.R.; SILVEIRA, M.L. Corporate values and stock performance of latin American banks. 2007. Social Science Research Network. Disponível em <www.ssrn.com>. Acesso em 01 de março de 2010.

ROSSI JUNIOR, J. L.; What is the value of corporate social responsibility? An answer from Brazilian sustainability index. Journal of International Business and Economics, 2009. Disponível em <www.ssrn.com>. Acesso em 08 de agosto de 2009.

TSAI, Chih-Chuan. The reputation effect and value in corporate social Responsibility. Unpublished Master Thesis, Department of Finance, Yuan Ze Unviersity, Taiwan, 2007. 